\title{
BREASTFEEDING PRACTICES AND LACTATION MASTITIS
}

\author{
Betsy Foxman, ${ }^{1}$ Kendra Schwartz ${ }^{2}$ and Sophia J. LoOMan ${ }^{3}$ \\ ${ }^{\text {'Department }}$ of Epidemiology, University of Michigan, Ann Arbor, MI 48109, U.S.A., ${ }^{2}$ Department of \\ Family Practice, Providence Hospital and ${ }^{3}$ Erasmus University, Rotterdam
}

\begin{abstract}
Clinical impression suggests that lactation mastitis is associated with inexperienced nursers, improper nursing techniques, stress and fatigue. A pilot study was conducted to describe the frequency of self-reported breastfeeding practices during the first week post partum among 100 breastfeeding women delivering at a freestanding birthing center or participating in an early discharge program. Nine cases of lactation mastitis were identified from the survey population and an additional 8 from the target population for the survey. Seventeen controls matched by delivery date were identified from survey participants. The frequency of self-reported breastfeeding practices, the presence of fatigue and stress during the week prior to the mastitis date in the case was compared among cases and controls. In the first week post partum, most women fed their babies every $2 \cdots 3 \mathrm{hr}$ for approx. $20 \mathrm{~min}$ a feeding. The cradle or Madonna position was the most frequently used nursing position. Nine percent reported supplementing feedings with formula. Women with mastitis were more likely than controls to report a history of mastitis with a previous child. In the week prior to the mastitis date of the case, women with mastitis were more likely than controls to report breast or nipple pain and cracks or breast fissures. They were less likely to report being able to take a daytime nap. Future studies should focus on the relative importance of and interrelationships among these factors.
\end{abstract}

Key words-lactation mastitis, breastfeeding, epidemiology

Puerperal or lactation mastitis is a cellulitis of the interlobular connective tissue within the mammary gland, with a clinical spectrum ranging from focal inflammation with minimal systemic symptoms to abscess and septicemia. The clinical definition includes fever of $38.5^{\circ} \mathrm{C}$ or greater, flu-like aches and chills, and an erythematous, tender, hot swollen wedge-shaped area of the breast [1]. The incidence of lactation mastitis has been reported to range from a low of $2.5 \%$, in a prospective study of women receiving care at a Health Maintenance Organization [2], to a high of $33 \%$ in a retrospective study of women participating in a breastfeeding conference who had breastfed in the previous 5 years [3].

Clinical impression suggests that lactation mastitis is associated with inexperienced nursers, improper nursing techniques [1], stress and fatigue [4]. However, a comprehensive review of the literature on lactation mastitis among humans over the past 50 years finds only 6 controlled studies published in English language journals examining risk factors and treatment for mastitis [2, 3, 5-8].

The most recent population-based study of the incidence of mastitis was conducted in 1945 in Great Britain, which reported an incidence of $8.9 \%$ [5]. Mastitis occurred more frequently among primiparae $(59 \%)$ compared to multiparae $(41 \%) ; 86 \%$ of the cases occurred before the end of the second week post-partum. More recently, Marshall et al. [2] reported the incidence among all women delivering infants and receiving post-partum care at the KaiserPermanente Medical System in Sacramento, California. The incidence among breast-feeding mothers was $2.5 \%$, with an average time until infection of 5.5 weeks. The nipple of the involved breast was fissured among $12 \%$ of the cases, but the rate of nipple fissures in the unaffected breast or among women without mastitis was not reported. Incidence by parity was also not reported. The most recent report was a descriptive, retrospective study among 180 women participating in conferences sponsored by the International Lactation Consultant Association and La Leche League International who had breastfed during the last 5 years [3]. Riordan et al. report a mastitis incidence of one third, where mastitis was defined as self-reported breast soreness and redness, flu-like aching and a fever above $100.4 \mathrm{~F}$. Mastitis was associated with fatigue, stress, plugged duct, change in number of feedings, engorgement/stasis, an infection in the family, breast trauma and poor diet. The effects of parity are not mentioned. About half the cases $(48 \%)$ occurred in the first 4 months post partum. This study was limited by its retrospective nature; the mastitis may have occurred as long as 5 years previously.

We conducted a record review study to describe the incidence of mastitis among breastfeeding women delivering at a single hospital [6]. Among the 972 women who were nursing upon discharge from the hospital between 1984 and 1985, the estimated 
incidence rate during the first 7 weeks following delivery was $4.4 / 1000$ women-weeks $(95 \% \mathrm{CI}$ : $2.61 / 1000$ to $6.1 / 1000$ women-weeks) or a 7 week risk of $3.0 \%$. Mastitis occurred most frequently among mothers aged $30-34$, even when the effects of parity and full-time employment outside the home were removed. Full-time employment outside the home was also associated with an increased risk of mastitis $(R R=2.65 ; 95 \% \mathrm{CI}: 0.9,7.7)$. Holding age constant, women without previous children were no more likely to have mastitis than women with one or more children $(R R=1.4 ; 95 \% \mathrm{CI}: 0.65,3.19)$.

It has been reported that improper breastfeeding techniques can lead to increased risk of mastitis, a failure to thrive in newborn infants, excessive concern and worry of breastfeeding mothers, and early weaning $[1,3,7,9,10]$. One breastfeeding technique, complete emptying of the breast, has been reported to improve outcome in women with mastitis $[7,8]$. Further, establishment of effective breastfeeding routines during the first few weeks post-partum are predictors of breastfeeding success [11]. If poor breastfeeding practices are truly a risk factor for the development of lactation mastitis and a poor outcome once diagnosed, we must know the frequency of these practices among breastfeeding women in order to develop appropriate interventions. We found no such descriptions even in select populations of breastfeeding women.

Published guidelines and recommendations for successful breastfeeding include a minimum of 8 feedings during a $24 \mathrm{hr}$ period with a range of $8-10$ most often advised $[1,10]$. Some authors recommend as many as 12 feedings per $24 \mathrm{hr}$ [12]. Five to $10 \mathrm{~min}$ of infant swallowing time per breast is recommended to assure adequate removal of milk from the breast [13]. Recommended positioning of the infant is variable, but all stipulate that the ventral surfaces of the mother and infant be opposed, whether the mother is laying, standing or sitting [13]. Recommendations for nipple care include the use of water only for cleaning, air drying after feeding, and breast pads to keep nipple dry [1].

In order to describe how frequently women follow recommended breastfeeding practices in the early post-partum period, we conducted a pilot study among breastfeeding women delivering at a freestanding birthing center or participating in an early discharge program. Women completed a selfadministered questionnaire regarding their recent breastfeeding practices. Within the same population we identified incident cases of lactation mastitis and compared them with controls matched on delivery date. Cases and controls completed a telephone interview regarding their breastfeeding practices during the week prior to the mastitis date of the case. Cases and controls were compared to determine the association of breastfeeding practices and other hypothesized risk factors such as fatigue and stress with lactation mastitis.

\section{METHODS}

\section{Breastfeeding survey}

Study participants were recruited from an early discharge program associated with a large teaching hospital and from a freestanding birthing center associated with a smaller community hospital. All breastfeeding mothers participating in the programs were invited to participate in the survey. Participants were given a questionnaire at discharge (freestanding birthing center) or on the third day postpartum (early discharge program). Questionnaires were self-administered and completed within the first 7 to 9 days postpartum. Approximately one-third of the women given questionnaires returned them, generally by mail.

The questionnaire asked about sociodemographic factors including age, marital status, parity, level of education and occupation of both parents, and household size. Breastfeeding questions included frequency and duration of feedings, position of infant, and changing breasts during feeding. Position of the infant during feeding was queried using drawing of the different positions. Madonna or cradle position is described as mother sitting with infant and mother's ventral surfaces opposed. The Australian is similar, but the mother is laying supine. Sidelying is lying, but side by side. In the football position, the infant is held laterally under just one breast (Fig. 1). Mothers were also asked about supplemental feeding, milk expression, engorgement, nipple pain and fluid consumption. Care of the breast and nipple was examined by asking about the use of nursing bras, pads, soap and topical medication. Fatigue was measured by questions about whether household help was available, the number of household duties of the mother, the amount of time the baby usually sleeps between feedings, if the mother is able to nap during the day, and total hours of sleep a day the mother has averaged since the baby's birth. The questionnaire was easily completed in $20 \mathrm{~min}$.

\section{Mastitis case finding}

Mastitis cases were identified from women who received obstetric care through either of two participating hospital clinics. Nine cases were identified among women participating in the survey described above. In addition, 8 women with mastitis who did not participate in the survey were referred by clinic staff. A case was defined as a woman reporting treatment for mastitis.

\section{Control selection}

Controls were selected from women participating in the survey of breastfeeding practices during the first week post partum. For each case, we identified all women who delivered within 6 weeks of the case's delivery date. Potential controls were ordered by time since delivery date of the case, from closest to furthest, and were contacted in order. We attempted to 
contact each potential control a minimum of five times. The first successful contact who was breastfeeding during the week prior to the date of mastitis diagnosis of the case and who had not developed mastitis while feeding their current baby was selected as the matched control.

\section{Mastitis questionnaire}

The questionnaire for both cases and controls was delivered in an approx. $30 \mathrm{~min}$ telephone interview. The content was similar to that of the breastfeeding survey, except all questions referred to the week prior to the mastitis date of the case. Additional questions were included to assess diet, stress, and symptoms (cases only) in the week prior to the mastitis date. Stress was measured using a short battery of good and bad events in the week prior to mastitis.

\section{Data analysis}

After coding and entry, data for both studies were analyzed using SAS [14].

For the analysis of the breastfeeding survey, we described the frequencies of variables using simple descriptive statistics and cross-tabulations. Associations between variables were measured using the

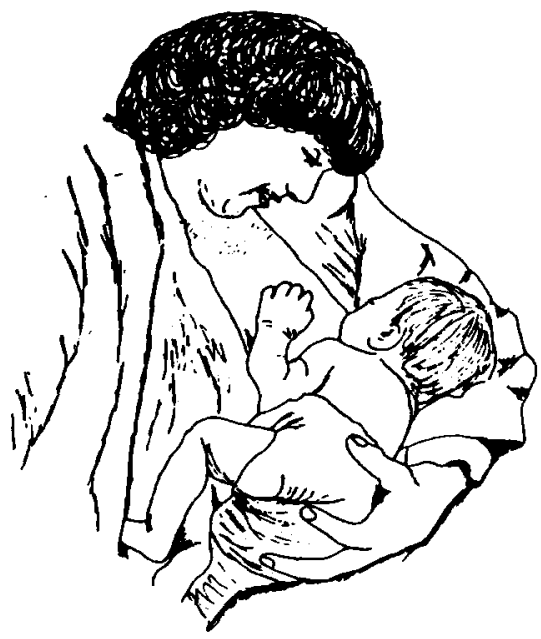

A) Cradle

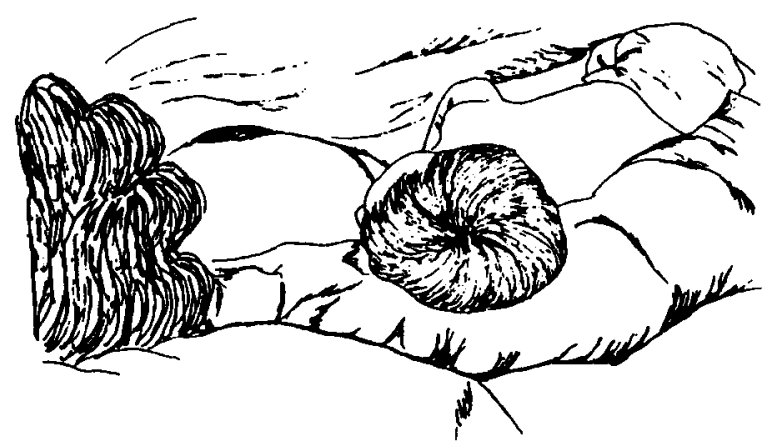

c) Sidelying odds ratio (OR) and $95 \%$ confidence intervals (95\% CI) and were tested using Student's $t$-test and $\chi^{2}$.

Data from the telephone survey of mastitis cases and controls were also described using simple descriptive statistics. We used a matched pair analysis to determine which categorical variables were associated with mastitis. The Odds Ratio (OR) and 95\% confidence intervals $(95 \% \mathrm{CI})$ were calculated using the MCSTRAT procedure found in SAS [14]. This procedure performs an iterative conditional maximumlikelihood fit of the logistic regression model. A separate model was run to determine the association of each variable with mastitis. For continuous variables we used a paired $t$-test. Because of the small sample size we did not attempt any multivariate analyses.

\section{RESULTS}

Characteristics of study population participating in breastfeeding survey

One hundred women, aged 19.41 $($ mean $=30.0$, STD 4.4) enrolled in the study. Most were White $(97 \%)$ and married $(96 \%)$. For about half of the women $(45 \%)$, this was their first experience with

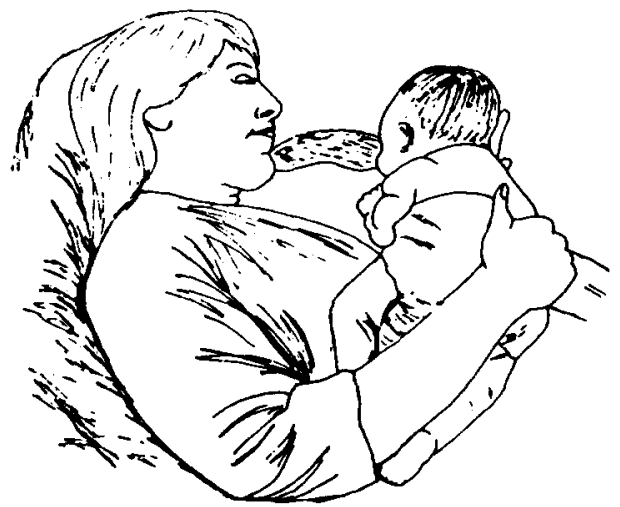

B) Australian

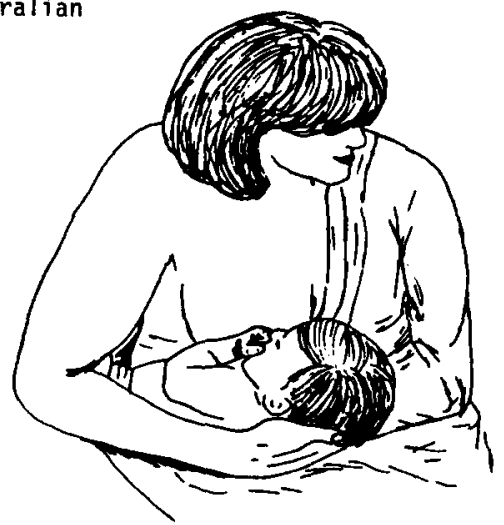

D) Football

Fig. 1. Breastfeeding positions. 
Table 1. Characteristics of 100 breastfeeding women. 1991

\begin{tabular}{|c|c|c|}
\hline Characteristic & N & $\%$ or Mean (STD) \\
\hline Average age & 100 & $30(4.4)$ \\
\hline \multicolumn{3}{|l|}{ Race,ethnicity } \\
\hline White & 97 & $(97)$ \\
\hline Other & 3 & $(3)$ \\
\hline \multicolumn{3}{|l|}{ Marital status } \\
\hline Married & 96 & $(96)$ \\
\hline Other & 4 & (4) \\
\hline \multicolumn{3}{|l|}{ Parity } \\
\hline 0 & 45 & (45) \\
\hline 1 & 27 & $(27)$ \\
\hline 2 & 15 & (15) \\
\hline 3 & 10 & (10) \\
\hline 4 or more & 3 & (3) \\
\hline \multicolumn{3}{|l|}{ Weaning age in months } \\
\hline Mother worked during pregnancy & 72 & $(72)$ \\
\hline \multicolumn{3}{|l|}{ If worked. hours worked } \\
\hline$\leqslant 20$ & 17 & $(19.7)$ \\
\hline 20.39 & 211 & $(2 \times .2)$ \\
\hline 40 or more & $3 i$ & $(521)$ \\
\hline \multicolumn{3}{|l|}{ Mother intends to return } \\
\hline \multicolumn{3}{|l|}{ Educational level. mother } \\
\hline Some high school & 1 & (1) \\
\hline High school degree & 22 & $(22.2)$ \\
\hline Some college & 22 & $(22.2)$ \\
\hline Bachelor's degree & 36 & $(36.4)$ \\
\hline Graduate education & 14 & $(14.1)$ \\
\hline Professional degree & 4 & (4) \\
\hline \multicolumn{3}{|l|}{ Educational level, father } \\
\hline Some high school & 2 & (2) \\
\hline High school degree & 14 & $(1.4 .3)$ \\
\hline Some collegc & 25 & $(25.5)$ \\
\hline Bachelor's degree & 37 & $(37 . x)$ \\
\hline Graduate educiation & 11 & $(11.2)$ \\
\hline Professional degree & y & (4) 2$)$ \\
\hline \multicolumn{3}{|l|}{ Occupation, nother } \\
\hline \multicolumn{3}{|l|}{ Professional. technical. } \\
\hline Clerical and sales & 15 & $(21.1)$ \\
\hline Other & 15 & $(21.1)$ \\
\hline \multicolumn{3}{|l|}{ Occupation, father } \\
\hline $\begin{array}{l}\text { Professionat technical. } \\
\text { or managerial }\end{array}$ & 6.5 & $(71.4)$ \\
\hline Clerical and sales & 4 & $(4.4)$ \\
\hline Other & 22 & $(24.2)$ \\
\hline
\end{tabular}

breastfeeding. About one third $(31 \%)$ with prior breastfeeding experience had a history of mastitis. The population was well educated: $54 \%$ held at least a bachelor" degree. Almost three-quarters (72\%) worked during pregnancy: of those who worked, most $(79 \%)$ planned to return to work within 6 months. All had health insurance which paid all or almost all of the costs of labor and delivery (Table 1).

Breastfeeding practices in the first weeks post-partum

Most women fed their babies every $2-3 \mathrm{hr}$ for approx. 20 min at feeding (Table 2). Over half (63\%) changed breasts during feeding. Although women experimented with several different nursing holds $174 \%$ (ried at least 3 holds), the cradle or Madonna position was most frequently or always used. Only 9 $(9 \%)$ reported supplementing feedings with formula (Table 2).

Over (wo-thirds $(69.7 \%)$ of study participants had experienced engorgement in the first week postpartum. About three-quarters $(74.7 \%)$ reported breast of nipple pain; almost three-quarters (71.2\%) had pain throughout nursing and one-fifth when the baby latched on $(21.9 \%)$. Only $13 \%$ used nipple medication- usually lanolin. Almost all wore a nursing bra $(98.9 \%)$. usually with breast pads $(69.3 \%)$ Nipple washing was relatively uncommon, either before $(14.1 \%)$ or after $(6.1 \%)$ feeding.

We hypothesized that we might find associations of sleep habits, mothers' education, use of breast pump, parity and nipple pain with either engorgement or formula supplementation. Although engorgement was associated with a negative answer to the question "Are you able to nap during the day," $(O R=1.85$; $95 \%$ CI: 0.54, 6.34), and formula supplementation with having at least some college $(\mathrm{OR}=2.47 ; 0.29$, $20.90)$ and using a breast pump $(\mathrm{OR}=6.9 ; 95 \% \mathrm{CI}$ :

Table 2. Breasteeding behaviors during the first week post partum $\left(A^{\prime}=100\right)$

\begin{tabular}{|c|c|c|c|}
\hline Behavior & $N$ & Meani $\pm S D !$ & Range \\
\hline Number of feedings each day & 90 & $8.61 \pm 2.11$ & 415 \\
\hline Feeding minutes each time & 96 & $23.21 \pm 8.4 i$ & 1055 \\
\hline \multirow[t]{2}{*}{ Average fluid consumption (k oz glasses) } & 98 & $8.6(+2.4)$ & 415 \\
\hline & $N$ & $"$ " & \\
\hline \multicolumn{4}{|l|}{ Positions frequently or always used } \\
\hline Cradle position & 89 & 89.11 & \\
\hline Sidelying position & 40) & 40.4 & \\
\hline Foothall position & 20 & 20.2 & \\
\hline Australian position & 10 & 10.4 & \\
\hline Usually wore a (nursing) bra & 88 & 98.9 & \\
\hline \multicolumn{4}{|l|}{ Switch breasts frequently or always } \\
\hline \multicolumn{4}{|l|}{ Wash nipples } \\
\hline Before leeding & 14 & 14.1 & \\
\hline After feeding & 6 & 6.1 & \\
\hline With soap & $\varphi$ & 9.1 & \\
\hline Used breastpump & 27 & 27.3 & \\
\hline Manual expression of milk & 40 & 40.8 & \\
\hline Any breast or nipple pain & 73 & 73.8 & \\
\hline Supplements with formula & 99 & 91 & \\
\hline
\end{tabular}


Table 3. Characteristics of lactation mastitis cases and controls matched ( $t 6$

\begin{tabular}{|c|c|c|}
\hline Characteristic & $\begin{array}{c}\text { Cases } \\
n(\%) \text { or mean (STD) }\end{array}$ & $\begin{array}{ll}\text { Controls } \\
n(\%) \text { or mean (STD) }\end{array}$ \\
\hline Average age & $30.2(4.9)$ & $29.7(4.4)$ \\
\hline Married & $14(93)$ & $16(94)$ \\
\hline White & $12(80)$ & $16(94)$ \\
\hline $\begin{array}{l}\text { At least some } \\
\text { college education }\end{array}$ & $13(93)$ & $12(75)$ \\
\hline $\begin{array}{l}\text { Number of children } \\
\text { in household }\end{array}$ & $2.7(1.45)$ & $2.0(0.94)$ \\
\hline First time mothers & $5(33)$ & $6(35)$ \\
\hline History of mastitis & $6(40)$ & $3(18)$ \\
\hline Baby's age (days) & $43.3(67.3)$ & $44.5(50.3)$ \\
\hline
\end{tabular}

Note: The denominator may be less than 17 due to missing values.

1.8, 26.1), most of the hypothesized relationships were only weakly associated and the confidence intervals were wide. This may reflect the relative homogeneity of our sample. The majority of study participants were committed to breastfeeding; in follow-up interviews most were still nursing at 3 months, and many at 6 months.

\section{Characteristics of mastitis cases and controls}

A total of 17 matched pairs were identified for study. Cases and controls were similar with respect to age, marital status, race, education and number of children (Table 3 ). The time since delivery was also similar. However, cases were more likely than controls to have previous children and to have a history of at least one occurrence of mastitis.

Mastitis occurred most frequently during the sixth week post-partum (range: 5-218 days). The most common symptoms were breast tenderness $(94 \%)$, malaise $(75 \%)$ and heat $(75 \%)$.

In the week prior to the date of mastitis, women with mastitis were more likely than controls to report any breast or nipple pain and cracks or breast fissures (Table 4). They were also more likely to report they could not take a daytime nap. Women with mastitis also reported fewer hours of sleep per day but the results failed to achieve conventional levels of significance (Table 5). Although cases had more children and had higher stress scores, they reported fewer household duties than controls. Neither of these results reach conventional levels of significance. Also of interest, women with mastitis averaged more frequent, shorter feedings and used a greater number of nursing positions (cradle, football, sidelying or Australian).

\section{DISCUSSION}

Studies of newborns at 5-7 days postpartum have shown that nutritive feeding time was 15 total minutes or less in $75 \%$ of feedings [15]. At 23.2 total minutes per feeding, participants in our breastfeeding survey tended to feed somewhat longer than physiologically necessary, and exceeded the minimum of 5-10 min of swallowing time per breast advised to assure adequate removal of milk from the breast [13]. However, nonnutritive sucking may be important in providing tactile stimulation to the nipple and areola, which is thought to promote the neurohormonal process of milk production [13]. Prolonged periods between feeding are thought to impair lactogenesis [10]. The average of 8.8 feedings observed in our sample compares favorably with the recommended a minimum of 8 feedings during a $24 \mathrm{hr}$ period [13].

Although women participating in our breastfeed. ing survey experimented with several nursing positions, the Madonna or cradle position was most frequently used. Changing positions is hypothesized to decrease nipple trauma by distributing the pressure more evenly around the areola [16]; however, we found no association between number of positions used and cracks and fissures.

There has been much controversy over the use of formula supplements early post-partum. For example, Lawrence has expressed concern that formula supplements will lead to early discontinuation

\begin{tabular}{|c|c|c|c|c|c|}
\hline Variable & $\begin{array}{l}\text { Cases } \\
n(\%)\end{array}$ & $\begin{array}{c}\text { Controls } \\
n(\%)\end{array}$ & OR $(95 \% \mathrm{CI})$ & $\chi^{2}$ & $P$-value \\
\hline \multicolumn{6}{|l|}{$\begin{array}{l}\text { Any breast or nipple } \\
\text { pain, in week prior }\end{array}$} \\
\hline \multicolumn{6}{|l|}{$\begin{array}{l}\text { Cracks or fissures } \\
\text { of breast, in week }\end{array}$} \\
\hline prior to diagnosis & $7(41.2)$ & $1(5.9)$ & $7.0(0.86,56.9)$ & 5.06 & 0.024 \\
\hline Ability to take daytime nap & $7(41.2)$ & $12(70.6)$ & $3.7 \times 10^{-5}(-,-)$ & 9.70 & 0.002 \\
\hline Use of breastpump & $3(17.6)$ & $6(35.3)$ & $0.4(0.08,2.06)$ & 1.33 & 0.249 \\
\hline History of previous mastitis & $6(40.0)$ & $3(17.6)$ & $5.0(0.58,42.48)$ & 2.91 & 0.088 \\
\hline
\end{tabular}


Table 5. Frequency of stress, breastfeeding and other maternal factors among women with lactation mastitis and matched control $(N=17)$ in the week prior to the mastitis date

\begin{tabular}{|c|c|c|c|c|}
\hline \multirow[b]{2}{*}{ Variable } & \multirow{2}{*}{$\begin{array}{c}\text { Cases } \\
\text { mean }( \pm S D)\end{array}$} & \multirow{2}{*}{$\begin{array}{c}\text { Controls } \\
\text { mean }( \pm S D)\end{array}$} & \multicolumn{2}{|c|}{ Paired } \\
\hline & & & $t$-value & $P$-value \\
\hline \multicolumn{5}{|l|}{ Maternal stress factors } \\
\hline Children in household & $2.7( \pm 1.45)$ & $2.0( \pm 0.94)$ & -1.53 & 0.14 \\
\hline Household duties & $3.2( \pm 3.24)$ & $4.4( \pm 2.20)$ & 1.40 & 0.17 \\
\hline Stress score & $0.9( \pm 1.81)$ & $0.4( \pm 0.61)$ & -1.26 & 0.22 \\
\hline \multicolumn{5}{|l|}{ Breastfeeding factors } \\
\hline Number of feedings/day & $8.0( \pm 2.50)$ & $7.4( \pm 3.12)$ & -0.63 & 0.53 \\
\hline Total minutes each feeding & $19.8( \pm 7.54)$ & $22.8( \pm 9.18)$ & 1.04 & 0.31 \\
\hline $\begin{array}{l}\text { Number of nursing positions } \\
\text { frequently or always used }\end{array}$ & $2.4( \pm 0.94)$ & $1.8( \pm 0.88)$ & -1.88 & 0.07 \\
\hline \multicolumn{5}{|l|}{ Other maternal factors } \\
\hline Glasses of fluid/day & $9.7( \pm 3.00)$ & $10.1( \pm 3.40)$ & 0.37 & 0.72 \\
\hline Hours sleep/day & $6.6( \pm 1.70)$ & $7.3( \pm 1.40)$ & 1.29 & 0.21 \\
\hline
\end{tabular}

of breastfeeding [1]. Almost $10 \%$ of the women in our breastfeeding survey used formula supplementation in the first week postpartum. Moreover, women with more education and who used a breastpump were more likely to supplement with formula. These findings and their impact on duration of breastfeeding need confirmation in a more representative sample of women.

Proper nipple care is purported to minimize the chance of nipple trauma [16]. Few of our survey participants washed their nipples either before or after feeding and even fewer used soap on their nipples; only a small percentage used nipple creams. Nursing bras with nursing pads were worn by almost all participants. In spite of this, nipple pain was reported by three-quarters of survey participants.

The incidence of mastitis among survey participants was $9 \%$, comparing favorably with the 1945 Great Britain population-based study report of $8.9 \%$ [5]. Comparing all mastitis cases to controls, we found the first evidence from a controlled study supporting conventional wisdom that breast cracks and fissures lead to mastitis. Women with mastitis also reported more breast and nipple pain in the week prior to mastitis than controls, but it is uncertain whether the pain was an early sign of mastitis or associated with a breast crack or fissure. Similarly, women with mastitis compared to controls reported using an increased number of nursing positions in the week prior to mastitis, but the increased number of positions may reflect the presence of breast cracks, fissures or pain rather than be a risk factor for mastitis. As was found in a recent study of mastitis [6], first time mothers had no greater risk of mastitis. Moreover, history of mastitis with a previous child was a risk factor, putting mothers with previous mastitis at an increased risk. The association of mastitis with not being able to take a daytime nap supports Lawrence's hypothesized association with fatigue [1].

\section{Limitations}

Our breastfeeding survey is the only report we know of where a relatively large number of women were asked to describe their breastfeeding practices in the first week post-partum. It should not, however, be taken as a definitive description because of several inherent limitations. Our participants are not representative of all breastfeeding women. Study participants were drawn from a pool of women who chose non-traditional delivery systems. Women who use these facilities for delivery are more likely to breastfeed; over $95 \%$ of women delivering at the freestanding birthing center breastfeed. Further, our response rate was low $(30 \%)$. Thus, we can not rule out that only women interested in and successful at breastfeeding participated. The breastfeeding practices described are only those in the first few weeks post partum, but establishment of good breastfeeding practices during this period is thought to be crucial for continued effective breastfeeding [11]. Although we did not actually observe the women breastfeeding, the self-administered questionnaire contained specific questions that covered only a recent time period. This should have increased the validity of the responses. Moreover, observation in itself can change behavior. Despite these limitations, the study does give information on how effective breastfeeders initially breastfeed as most of the women breastfed for more than 3 months. Larger studies in a representative sample of women who breastfeed are in order.

The small sample size of the case control study also limits our ability to generalize and make definitive conclusions. We were unable, for example, to identify or control the effects of third variables on the observed associations. Moreover, our study population was predominantly White and of upper socioeconomic status: all had insurance which covered all or almost all their costs of labor and delivery. Nonetheless the study gives us insight into previously unexplored associations between lactation mastitis and potential risk factors.

Because cases and controls were queried about the week prior to the mastitis date of the case, it is possible that cases were more likely to recall their exposure to factors of interest than controls. Recall bias most frequently occurs when study hypotheses are know to study participants or are widely discussed in the lay literature. Neither of factors are true in this case, thus we believe a recall bias is unlikely. 


\section{Conclusions}

Studies are needed to verify that recommended breastfeeding practices lead to successful breastfeeding. It would then be possible to identify the most successful initial breastfeeding habits to clinically as well as physiologically define recommendations for effective breastfeeding. A larger study of a more representative group is necessary to explore the associations of breastfeeding practices, lactation mastitis and duration of breastfeeding. Based on this pilot case control study, future studies of mastitis should examine factors leading to breast cracks and fissures, and the role of mastitis history, number of nursing positions and amount of sleep on risk of mastitis.

Acknowledgements - This work was supported in part by Providence Hospital. The authors thank Patricia Hogan, Margaret Hanson, and Ronald Burkman of the Henry Ford Great Expectations Program and Mary Lou Longeway and staff at Providence Family Birthing Center for their assistance.

\section{REFERENCES}

1. Lawrence R. A. Breastfeeding: A Guide for the Medical Profession. C. V. Mosby, Saint Louis, 1989.

2. Marshall B. R., Hepper J. K. and Zirbel C. C. Sporadic puerperal mastitis: An infection that need not interrupt lactation. JAMA 233, 1377-1379, 1975

3. Riordan J. M. and Nichols F. H. A descriptive study of lactation mastitis in long-term breastfeeding women. J. Human Lact. 6, 53-58, 1990.
4. Applebaum R. M. The modern management of successful breast feeding. Pediatr. clin. N. Am. 17, 203-225, 1970.

5. Fulton A. A. Incidence of puerperal and lactational mastitis in an industrial town of some 43,000 inhabitants. Br. Med. J. 1, 693-696, 1945.

6. Kaufmann R. and Foxman B. Mastitis among lactating women: occurrence and determinants. Soc. Sci. Med. 33, 701-705, 1991 .

7. Thomsen A. C., Espersen T. and Maigaard S. Course and treatment of milk stasis, noninfectious inflammation of the breast, and infectious mastitis in nursing women. Am. J. Obstet. Gynecol. 149, 492 495, 1984.

8. Matheson I., Aursnes I., Horgen M., Aabo O. and Melby $K$. Bacteriological findings and clinical symptoms in relation to clinical outcome in puerperal mastitis. Acta. Obst. Gyn. Scand. 67, 723-726, 1988.

9. Niebyl J. R., Spence M. R. and Parmley T. H. Sporadic (nonepidemic) puerperal mastitis. J. Reprod. Med. 20, 97-100, 1978.

10. Neifert M. R. and Seacat J. M. A guide to successful breast-feeding. Contemp. Pediat. 3, 26-45, 1986.

11. Matthews M. K. Mothers' satisfaction with their neonates breastfeeding behavior. JOGNN 20, 49-55, 1991.

12. Klaus M. H. The frequency of suckling a neglected but essential ingredient of breast-feeding. Obstet. Gynecol. clin. N. Am. 14, 623-633, 1987.

13. Shrago $L$. and Bocar $D$. The infant's contribution to breastfeeding. JOGNN 19, 209-215, 1990.

14. SAS Institute Inc. SAS, Version 5.16 (c) 1986 SAS Institute, Inc., Cary, N.C.

15. Howie P. W., Houston M. J., Cook A., Smart L., McArdle T. and McNeilly A. S. How long should a breast feed last? Early Hum. Devel. 5, 71-77, 1981.

16. L'Esperance $\mathrm{C}$. and Frantz $\mathrm{K}$. Time limitation for early breastfeeding. JOGNN 14, 114-118, 1985. 